\title{
Ultrasound-guided cannulation of the left subclavian vein in a case of persistent left superior vena cava
}

\author{
Diane Commandeur, MD - Marc Garetier, MD - Christophe Giacardi, MD • \\ Sophie Huynh, MD · Marc Danguy des Deserts, MD • Marie-Laure Buguet-Brown, MD • \\ Mehdi Ould-Ahmed, MD · Jean Rousset, MD
}

Received: 14 December 2010/ Accepted: 31 January 2011/Published online: 19 February 2011

(C) Canadian Anesthesiologists' Society 2011

Ultrasound guidance is useful for venipuncture and placement of central venous access catheters; however, anatomical variants may complicate catheter insertion.

A 52-yr-old female patient was admitted to the intensive care unit with acute respiratory distress due to bilateral pneumonia. Her respiratory status worsened rapidly and she required mechanical ventilation. For cardiovascular monitoring, an ultrasound-guided cannulation of her left subclavian vein was performed uneventfully using an Acuson $\mathrm{CV} 70^{\mathrm{TM}}$ cardiovascular ultrasound system (Siemens ${ }^{\circledR}$, Erlangen, Germany), and a central venous catheter (CVC) was inserted. A chest $x$-ray revealed a left-sided paramediastinal course of the CVC (Figure, Panel A), at which point, we considered the potential for arterial placement, accidental venous placement in normal structures (superior intercostal vein or internal thoracic vein), or a malformed left superior vena cava. Subsequently, the diagnosis of persistent left superior vena cava (PLSVC) was confirmed by thoracic computed tomography with contrast injection. Reconstructed computer tomography scan imaging (Figure, Panel B) showed the left subclavian vein give rise to the PLSVC, which drains into in the left atrium, and the brachiocephalic vein that forms the right superior vena cava.
The CVC was then removed, and another was inserted uneventfully into the right internal jugular vein using ultrasound guidance. The patient's respiratory status improved progressively, and her trachea was extubated 21 days later.

Persistent left superior vena cava is the most common major anomaly of the systemic venous system. This anatomic variant, which is asymptomatic, results from persistence of an embryological vessel that normally involutes into the coronary sinus. Persistent left superior vena cava is not an absolute contraindication for subclavian vein cannulation if adequate drainage into the right atrium exists. Nevertheless, difficulties can arise during CVC insertion, transjugular intrahepatic portosystemic shunt insertion, and pulmonary artery catheter and pacemaker insertion. The etiology of the CVC false tracking in patients with PLSVC is usually established by chest $x$-ray and can be confirmed by echocardiography. This case illustrates that thoracic computed tomography is an important diagnostic imaging modality to delineate variant central venous anatomy.

Competing interests None declared.
D. Commandeur, MD $(\bowtie) \cdot$ M. Garetier, MD .

C. Giacardi, MD - S. Huynh, MD - M. D. d. Deserts, MD .

M.-L. Buguet-Brown, MD · M. Ould-Ahmed, MD .

J. Rousset, MD

Military Teaching Hospital "Clermont-Tonnerre", Brest, France

e-mail: diane.commandeur@wanadoo.fr 
Figure Panel a Chest $x$-ray following central venous catheter (CVC) insertion showing the left-sided paramediastinal course of the CVC (1). Panel b Reconstructed computer tomography scan image of the heart and great vessels showing: 1) axillary vein, 2) scapular vein, 3) left subclavian vein, 4) persistent left superior vena cava, 5) brachiocephalic vein, and 6) right superior vena cava
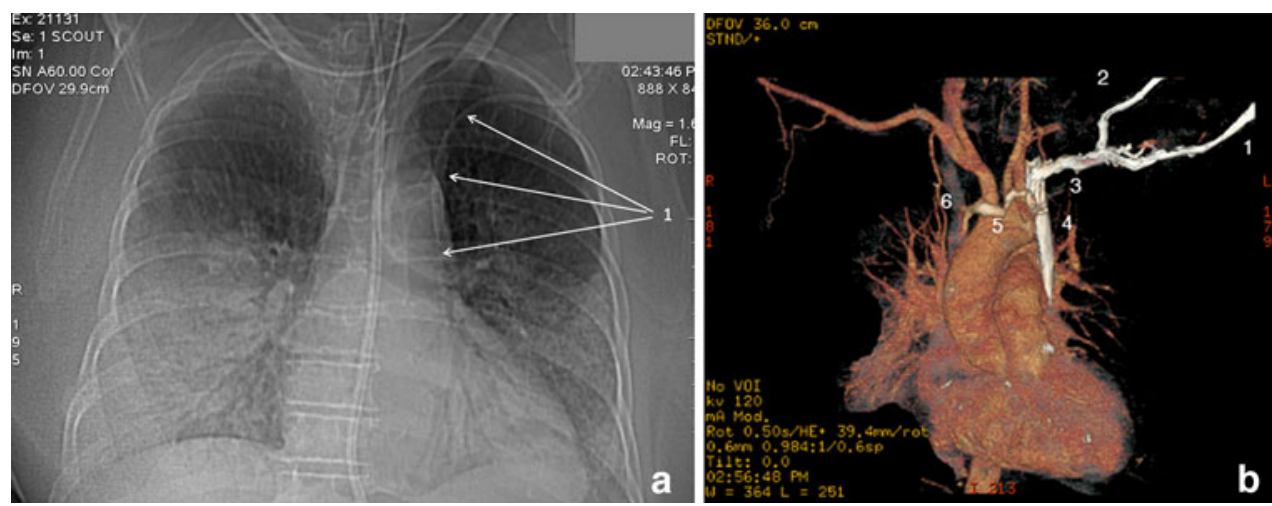